UNF-Astro-3-1-10C

\title{
SENSITIVITY OF THE FERMI DETECTORS TO GAMMA-RAY BURSTS FROM EVAPORATING PRIMORDIAL BLACK HOLES (PBHS)
}

\author{
T. N. UKWATTA ${ }^{1,2}$, J. H. MACGIBBON ${ }^{3 *}$, W. C. PARKE ${ }^{1}$, K. S. DHUGA ${ }^{1}$, S. RHODES $^{3}$, \\ A. ESKANDARIAN ${ }^{1}$, N. GEHRELS ${ }^{2}$, L. MAXIMON $^{1}$, and D. C. MORRIS ${ }^{1,2}$ \\ ${ }^{1}$ The George Washington University, \\ Washington, D.C. 20052, USA \\ 2 NASA Goddard Space Flight Center, \\ Greenbelt, MD 20771, USA \\ ${ }^{3}$ Department of Physics, University of North Florida, \\ Jacksonville, FL 32224, USA \\ *E-mail: jmacgibb@unf.edu
}

\begin{abstract}
Primordial Black Holes (PBHs), which may have been created in the early Universe, are predicted to be detectable by their Hawking radiation. The Fermi Gamma-ray Space Telescope observatory offers increased sensitivity to the gamma-ray bursts produced by PBHs with an initial mass of $\sim 5 \times 10^{14} \mathrm{~g}$ expiring today. PBHs are candidate progenitors of unidentified Gamma-Ray Bursts (GRBs) that lack X-ray afterglow. We propose spectral lag, which is the temporal delay between the high and low energy pulses, as an efficient method to identify PBH evaporation events with the Fermi Large Area Telescope (LAT).
\end{abstract}

Keywords: Gamma-Ray Bursts; Primordial Black Holes; Spectral Lag.

\section{Introduction}

The formation of Primordial Black Holes (PBHs) has been postulated in many theories of the early Universe (for a recent review see Ref. 1). Black holes of mass $M_{\mathrm{bh}}$ continually emit Hawking radiation ${ }^{2}$ with a temperature of $T_{\mathrm{bh}}=$ $1.06 \mathrm{GeV} /\left(M_{\mathrm{bh}} / 10^{13} \mathrm{~g}\right)$ in the form of all available fundamental particle species. The emitted particles decay quickly on astrophysical timescales into $\gamma, \nu_{e, \mu, \tau}, \bar{\nu}_{e, \mu, \tau}$, $p, \bar{p}, e^{+}$and $e^{-}$. PBHs with an initial mass ${ }^{3}$ of $M_{*} \sim 5 \times 10^{14} \mathrm{~g}$ should be expiring today with a burst of high energy particles including gamma-rays. The current upper limit on the number expiring today per volume per unit time is $\underline{4}$

$$
R \lesssim 10^{-7} \eta_{\text {local }} \mathrm{pc}^{-3} \mathrm{yr}^{-1}
$$

where $\eta_{\text {local }}$ is the density enhancement of PBHs in the local region. Typically $\eta_{\text {local }}$ is $\sim 10^{6}$ (for clustering in the Galactic halo) or larger. Such PBH bursts may be detectable by the Fermi Gamma-ray Space Telescope observatory's Large Area Telescope (LAT). Conversely, non-detection by the LAT may lead to tighter bounds on the $\mathrm{PBH}$ distribution. 


\section{PBH burst as seen by Ideal Detector}

In the standard mode ${ }^{3}$, the total number of photons emitted per second by a $T_{\mathrm{bh}} \sim 0.3-100 \mathrm{GeV}$ black hole scales as ${ }^{5}$

$$
\dot{N}_{\mathrm{bh} \gamma} \simeq 1.4 \times 10^{29}\left(\frac{T_{\mathrm{bh}}}{\mathrm{TeV}}\right)^{1.6} \mathrm{~s}^{-1} .
$$

The number of photons per second per unit area reaching the Earth from a PBH bursting at a distance $d$ from the Earth is

$$
F_{\mathrm{bh}}=\frac{\dot{N}_{\mathrm{bh} \gamma}}{4 \pi d^{2}}
$$

Let us assume an ideal detector of effective area $A_{\text {eff }}$ which can detect every photon that falls on it. (If the detector is non-ideal then the efficiency can be incorprated into the value of $A_{\text {eff. }}$.) If the detector requires $X$ photons over time $t$ to distinguish an incoming event as a burst, then to detect a burst we require $F_{\mathrm{bh}} A_{\text {eff }} t \geq X$. That is the $\mathrm{PBH}$ must be closer than

$$
d_{\mathrm{D}} \simeq \frac{2.6 \times 10^{-2}}{\sqrt{X}}\left(\frac{T_{\mathrm{bh}}}{\mathrm{TeV}}\right)^{0.8}\left(\frac{A_{\mathrm{eff}}}{\mathrm{m}^{2}}\right)^{1 / 2}\left(\frac{t}{\mathrm{~min}}\right)^{1 / 2} \mathrm{pc} .
$$

What $T_{\mathrm{bh}}$ maximizes the chance of detection? The remaining lifetime ${ }^{6}$ of a $\mathrm{PBH}$ of temperature $T_{\mathrm{bh}}$ is $\tau_{\text {evap }} \simeq 7.4 \times 10^{3} /\left(T_{\mathrm{bh}} / \mathrm{TeV}\right)^{3} f \mathrm{~s}$ where $f\left(T_{\mathrm{bh}}\right)$ weights the number of emitted species. (The remaining lifetime of a $300 \mathrm{GeV}, 1 \mathrm{TeV}$, or $5 \mathrm{TeV}$ black hole is 1 hour, 1 minute, or 1 second, respectively.) Taking $t=\tau_{\text {evap }}$, a PBH will be detected by the ideal detector if it is closer than

$$
d_{\mathrm{D}} \simeq \frac{0.03}{\sqrt{X}}\left(\frac{T_{\mathrm{bh}}}{\mathrm{TeV}}\right)^{-0.7}\left(\frac{A_{\mathrm{eff}}}{\mathrm{m}^{2}}\right)^{1 / 2} \mathrm{pc}
$$

Thus the detectability is maximized for the lowest $T_{\mathrm{bh}}$ black hole visible above the background and/or by using the longest detector exposure time. For a detector of angular resolution $\Omega$ to resolve the $\mathrm{PBH}$ above the gamma-ray background $F_{\gamma}$, we also require that $F_{\mathrm{bh}} A_{\text {eff }} \geq F_{\gamma} \Omega A_{\text {eff }}$. The $\mathrm{PBH}$ will be resolved above the observed (EGRET) extragalactic background ${ }^{7}$

$$
\frac{d F_{\gamma}}{d E} \simeq 1.4 \times 10^{-6}\left(\frac{E}{\mathrm{GeV}}\right)^{-2.1} \mathrm{~cm}^{-2} \mathrm{GeV}^{-1} \mathrm{~s}^{-1} \mathrm{sr}^{-1}
$$

at energy $E$ by the ideal detector if the $\mathrm{PBH}$ is closer than

$$
d_{\mathrm{R}} \simeq 0.03\left(\frac{\Omega}{\mathrm{sr}}\right)^{-1 / 2}\left(\frac{E}{\mathrm{GeV}}\right)^{0.55}\left(\frac{T_{\mathrm{bh}}}{\mathrm{TeV}}\right)^{0.8} \mathrm{pc}
$$

and $E$ is less than the average energy ${ }^{5}$ of the PBH photons $\bar{E}_{\gamma} \approx 10\left(T_{\mathrm{bh}} / \mathrm{TeV}\right)^{0.5}$ $\mathrm{GeV}$. The isotropic diffuse gamma-ray background, which is an upper limit on the extragalactic background, recently measured ${ }^{8}$ by the LAT at mid-Galactic latitudes is consistent with the earlier EGRET measurements Eq. (6), although the extragalactic component may ${ }^{\sqrt{9}}$ be weaker by up to a factor of 2 . 
For a given detector, the scanned volume of space is then $V_{\mathrm{bh}}=\left(\omega_{\mathrm{A}} / \mathrm{sr}\right) d_{\mathrm{S}}^{3} / 3$ where $\omega_{\mathrm{A}}$ is the detector acceptance angle (field of view) and $d_{\mathrm{S}}=\min \left(d_{\mathrm{D}}, d_{\mathrm{R}}\right)$. Extensive air shower arrays characteristically have $A_{\text {eff }} \gtrsim 10^{4} \mathrm{~m}^{2}$, large $\omega_{\mathrm{A}}$ and small $\Omega$ but very high threshold energy (typically $\gtrsim 10 \mathrm{TeV}$ ) and hence are background-limited, while atmospheric Cerenkov detectors 10 characteristically have $A_{\text {eff }} \gtrsim 200 \mathrm{~m}^{2}$ and small $\Omega$ but high threshold energy (typically $\gtrsim 100 \mathrm{GeV}$ although the Whipple SGARFACE system $\frac{111}{10}$ has a threshold of $100 \mathrm{MeV}$ ) and very small $\omega_{\mathrm{A}}$ $\left(\lesssim 10^{-2} \mathrm{sr}\right)$. In contrast, the Fermi LAT has $\frac{12}{12}$ a smaller $A_{\text {eff }} \sim 0.8 \mathrm{~m}^{2}$ but large $\omega_{\mathrm{A}} \sim 2.4 \mathrm{sr}$, finer source position angular resolution $\left(0.3-2^{\prime}\right)$, low energy thresholds (down to $20 \mathrm{MeV}$ ), good time resolution and is essentially background-free with respect to burst sensitivity. Additionally, most of the photons emitted by expiring $T_{\mathrm{bh}} \lesssim 1 \mathrm{TeV}$ PBHs are in the LAT energy range $(20 \mathrm{MeV}-300 \mathrm{GeV})$.

\section{Conclusions}

The Fermi LAT offers greater sensitivity to local PBH bursts than ground-based detectors. We have proposed ${ }^{13}$ spectral lag measurements (the temporal delay between high and low energy pulses) of the incoming light curve in two different energy bands as a method to identify PBH bursts. A PBH burst arriving at the detector will exhibit positive to negative evolution with increasing energy because the black hole temperature and $\bar{E}_{\gamma}$ increase over time as the black hole loses mass. Because spectral lag measurements require counts in only two energy bands, and not the full spectrum, spectral lag can be measured even for weak events that last for very short time scales. Work is in progress to calculate quantitative values for the $\mathrm{PBH}$ spectral lags for the characteristics of the Fermi LAT.

\section{References}

1. B. J. Carr, in Inflating Horizons of Particle Astrophysics and Cosmology, (Universal Academy Press Inc, Tokyo, 2006).

2. S. W. Hawking, Nature 248, 30 (1974); S. W. Hawking, Commun. Math. Phys. 43, 199 (1975); S. W. Hawking, Phys. Rev. D 13, 191 (1976).

3. J. H. MacGibbon, B. J. Carr, and D. N. Page, Phys. Rev. D 78, 064043 (2008).

4. J. H. MacGibbon and B. J. Carr, Astrophys. J. 371, 447 (1991).

5. J. H. MacGibbon and B. R. Webber, Phys. Rev. D 41, 3052 (1990).

6. J. H. MacGibbon, Phys. Rev. D 44, 376 (1991).

7. P. Shreekumar et al., Astrophys. J. 494, 523 (1998).

8. A. A. Abdo et al., Astrophys. J. 703, 1249 (2009).

9. A. W. Strong, I. V. Moskalenko, and O. Reimer, Astrophys. J. 613, 956 (2004).

10. L. A. Antonelli et al., The Next Generation of Cherenkov Telescopes. A White Paper for the Italian National Institute for Astrophysics (INAF) arXiv:0906.4114.

11. S. LeBohec, F. Krennrich, and G. Sleege Astropart. Phys. 23, 235 (2005).

12. W. B. Atwood et al., The Large Area Telescope on the Fermi Gamma-ray Space Telescope Mission arXiv:0902.1089.

13. T. N. Ukwatta et al., AIP Conf. Proc. 1133, 440 (2009). 